\title{
Evaluating molecular modeling tools for thermal stability using an independently generated dataset
}

\author{
Peishan Huang, 1 Simon K. S. Chu, 1 Henrique N. Frizzo,2 Morgan P. Connolly,3 Ryan W. Caster,2 Justin B. Siegel2,4,5,*
}

1Biophysics Graduate Group, University of California, Davis, California, United States of America

${ }_{2}$ Genome Center, University of California, Davis, California, United States of America

3Microbiology Graduate Group, University of California, Davis, California, United States of America

4Department of Biochemistry \& Molecular Medicine, University of California, Davis, California, United States of America, ${ }_{5}$ Department of Chemistry, University of California, Davis, California, United States of America

KEYWORDS Thermal stability, Rosetta Molecular Modeling Suite, molecular modeling, Protein Engineering

\begin{abstract}
Engineering proteins to enhance thermal stability is a widely utilized approach for creating industrially relevant biocatalysts. Computational tools that guide these engineering efforts remain an active area of research with new data sets and develop algorithms. To aid in these efforts, we are reporting an expansion of our previously published data set of mutants for a $\beta$-glucosidase to include both measures of $\mathrm{T}_{\mathrm{M}}$ and $\Delta \Delta \mathrm{G}$, to complement the previously reported measures of $\mathrm{T}_{50}$ and kinetic constants $\left(k_{\mathrm{cat}}\right.$ and $\left.K_{\mathrm{M}}\right)$. For a set of 51 mutants, we found that $\mathrm{T}_{50}$ and $\mathrm{T}_{\mathrm{M}}$ are moderately correlated with a Pearson correlation coefficient (PCC) of 0.58 , indicated the two methods capture different physical features. The performance of predicted stability using five computational tools are also evaluated on the 51 mutants dataset, none of which are found to be strong predictors of the observed changes in $\mathrm{T}_{50}$, $\mathrm{T}_{\mathrm{M}}$, or $\Delta \Delta \mathrm{G}$. Furthermore, the ability of the five algorithms to predict the production of isolatable soluble protein is examined, which revealed that Rosetta $\Delta \Delta \mathrm{G}$, ELASPIC, and DeepDDG are capable of predicting if a mutant could be produced and isolated as a soluble protein. These results further highlight the need for new algorithms for predicting modest, yet important, changes in thermal stability as well as a new utility for current algorithms for prescreening designs for the production of soluble mutants.
\end{abstract}

\section{INTRODUCTION}

A common goal of enzyme engineering is the enhancement of thermal stability.1 For industrial applications improving a proteins' robustness to thermal challenges or half-life at elevated temperature can often be the deciding factor for the commercialization of a biocatalyst.2-5 Currently, the most common approach for improving thermal stability is through directed evolution methodologies, 6,7 which, can be timeconsuming, costly, and limited in the ability to search sequence space. Computational design tools to predictably identify mutations, single and combinatorial that enhance thermal stability are rapidly developing and growing in popularity.8-11 However, accurate predictions using computational tools to guide protein stability design remains an active area of research and is not always successful.

The use of large data sets on the mutational effect on protein stability, such as ProTherm, is often used to train computational methods for predicting thermal stability. The data sets utilized generally composed of the equilibrium constant of unfolding $(\mathrm{KU})$ or the melting temperature of an enzyme (TM).12 In our previous study, we determined the thermal stability of $79 \beta$-glucosidase B (BglB) variants by finding T $50-$ a type of kinetic stability that is determined by the temperature at which a mutant's residual activity is reduced by $50 \%$ after a heat-challenge over a defined time.4,12,13 When analyzing this set of mutants using two established computational programs (Rosetta $\Delta \Delta \mathrm{G}$ and FoldX PSSM) for predicting thermal stability, we found that there was no significant correlation between the predictions and the observed T50.14

One hypothesis explaining the poor predictive performance of the algorithms with the $\mathrm{BglB}$ dataset is that the algorithms are trained on $\mathrm{T}_{\mathrm{M}}$, a direct measure of structural thermal stability, but being used to predict $\mathrm{T}_{50}$, an indirect measure of the protein's thermal stability.12 Alternatively, the poor performance could have come from the narrow $\mathrm{T}_{50}$ range (extreme variants are $+6.06{ }^{\circ} \mathrm{C}$ and $-5.02{ }^{\circ} \mathrm{C}$ from wild type), as the algorithms are generally trained on larger changes in thermal stability and $\pm 5{ }^{\circ} \mathrm{C}$ may be within the error of the currently developed algorithms. In this study, we evaluated both hypotheses. To assess if there is a significant difference in $\mathrm{T}_{\mathrm{M}}$ and $\mathrm{T}_{50}$ we developed a data set of $51 \mathrm{BglB}$ mutants (Figure 1) in which both thermal stability measurements, $\mathrm{T}_{50}$ and $\mathrm{T}_{\mathrm{M}}$, are measured.

Interestingly, for the set of 51 measurements, there is only a modest correlation between $\mathrm{T}_{50}$ and $\mathrm{T}_{\mathrm{M}}$, with a PCC of 0.58 . This highlights the difference in the physical properties being measured using these two techniques, $\mathrm{T}_{\mathrm{M}}$ being the thermal 
stability of the protein's structural elements and T50 reporting on the thermal stability to irreversible denaturation. However, similar to the previous study,14 the relationship between the predicted stability with the experimental $T_{M}$ only results in a weak correlation not only with the previous algorithms evaluated (Rosetta $\Delta \Delta \mathrm{G}$ and FoldX PSSM) but also three other commonly used methods: ELASPIC, DeepDDG, and PoPMuSiC. This result suggests that while the two measurements are reporting on different physical properties, this is not the key factor that led to the low predictive accuracy of established algorithms on this data set.

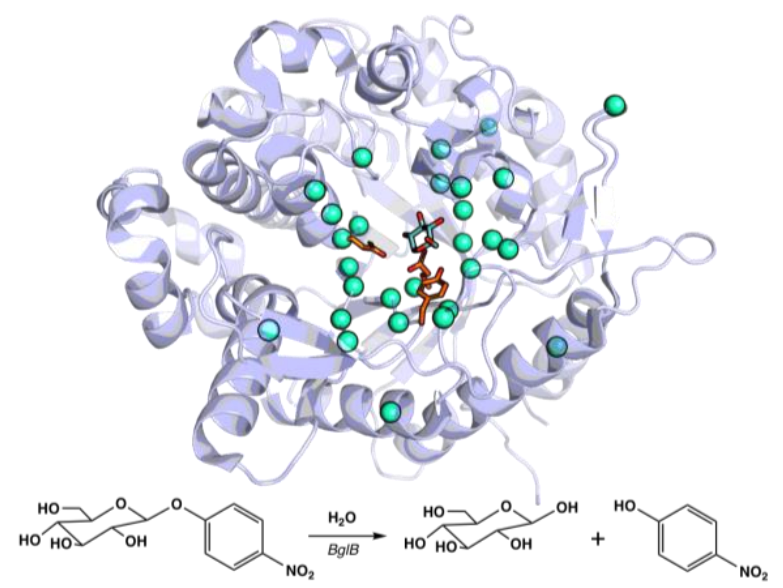

Figure 1. Structure of BglB (PDB ID: 2JIE) from bacterium Paenibacillus polymyxa. PyMOL rendering 15 of BglB showing the 28 sequence-positions (teal spheres) of the 51 mutants randomly selected for $\mathrm{T}_{\mathrm{M}}$ analysis. The reaction scheme of the hydrolysis of 4-nitrophenyl $\beta$-D-glucopyranoside by BglB used in the T50 study. 14

To evaluate the second hypothesis, that the changes in thermal stability of the BglB data set are too small for current algorithms, we investigated the ability of the algorithms to predict soluble protein production. Mutations that reduce thermal stability (TM) by $>18{ }^{\circ} \mathrm{C}$ also fail to yield soluble, isolatable protein. Analysis of computational algorithms enriched for insoluble mutants based on the predicted energetics showed a significant enrichment was observed for Rosetta $\Delta \Delta \mathrm{G}$. This supports the hypothesis that the lack of performance on the $\mathrm{BglB}$ data set is due to the narrow range in thermal stability changes observed. This highlights the need for new algorithms for predicting modest, yet important, changes in thermal stability as well as a new utility for current algorithms for prescreening designs for the production of soluble protein.

\section{METHODS}

Mutant Selection, protein expression, and purification. Out of 79 mutants of Beta-glucosidase $\mathrm{B}(\mathrm{BglB})$ that were previously characterized with $\mathrm{T}_{50}$ data, 1451 variants with plasmid readily available were transformed into chemically competent $E$. coli BLR(DE3) cells. The variants were produced and purified as previously described.14 Expression was carried out by growing a $5 \mathrm{~mL}$ overnight culture in a $50 \mathrm{~mL}$ falcon tube with breathable seal in Terrific broth (TB) medium with kanamycin while shaking at $250 \mathrm{rpm}$ at $37^{\circ} \mathrm{C}$. After the initial overnight culture cells were spun down and resuspended in fresh TB with kanamycin with $1 \mathrm{mM}$ Isopropyl $\beta$-D-1thiogalactopyranoside (IPTG) in a $50 \mathrm{~mL}$ falcon tube with breathable seal and incubated while shaking at $250 \mathrm{rpm}$ at $18 \mathrm{C}$ for 24 hours. Then the cells were then spun down, lysed, and purified samples using immobilized metal ion affinity chromatography as previously described.14 The purity of the protein samples was analyzed using 12-14\% SDS-PAGE (SI 11 ), the yield was assessed based on the A280 for proteins that appeared $>75 \%$ pure in the SDS-PAGE analysis.

Melting Temperature assay. The melting temperature (TM) of BglB was determined using the Protein Thermal Shift (PTS) TM kit (Applied BioSystem ®, from Thermo Fisher). Standard protocols provided by the manufacturer were used. Protein concentrations ranged from $0.1-0.5 \mathrm{mg} / \mathrm{mL}$ and fluorescence reading was monitored with a QuantaStudio ${ }^{\mathrm{TM}} 3$ System from $20{ }^{\circ} \mathrm{C}$ to $90{ }^{\circ} \mathrm{C}$. The temperature melting curve was first smoothened with a 20 -step sliding window average (SI 4). TM was determined from the average of 4 replicates at which the derivative was largest, and all melting curves can be found in SI 2.

$\Delta \Delta \mathbf{G}$ Calculations from $\mathbf{T}_{\mathbf{M}}$. Calculations were carried out in a similar manner as previously described.15 Briefly, to derive $\Delta \mathrm{G}^{\circ}$ unfolding the fluorescence intensity was first translated into fraction of folded $\left(\mathrm{P}_{\mathrm{f}}\right)$ and unfolded $\left(\mathrm{P}_{\mathrm{u}}\right)$ proteins at different temperatures starting from the minimum fluorescence $\left(F_{\min }\right)$ to the maximum fluorescence $\left(F_{\max }\right)$ shown in eq 1 .

$$
P_{f}=1-\frac{F-F_{\min }}{F_{\max }-F_{\min }}
$$

By taking a two-state folding-unfolding model, the equilibrium constant of unfolding $\left(K_{\mathrm{u}}\right)$ at different temperatures is then given by:

$$
K_{u}=\frac{P_{u}}{P_{f}}
$$

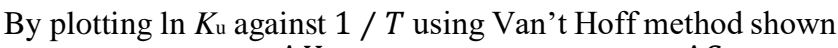
in eq 3 (SI 2), the $-\Delta H /_{R}$ is defined by the slope, $\Delta S /_{R}$ is the $\mathrm{y}$-intercept, $\mathrm{T}$ is temperature, and $R$ is the ideal gas constant.

$$
\ln K_{u}=-\frac{\Delta H}{R T}+\frac{\Delta S}{R}
$$

The Gibbs free energy of protein-unfolding ( $\Delta \mathrm{G}^{\circ}$ unfolding) can then be determined using eq 4 , where $\Delta \mathrm{G}^{\circ}$ unfolding is the unfolding energy at $298 \mathrm{~K}$ as T. All calculations can be found under SI 4.

$$
\Delta G^{\circ}{ }_{\text {unfolding }}=\Delta H-T \Delta S
$$

Molecular modeling. Five popular, readily accessible, and recently developed force-field and machine-learning-based molecular modeling methods were evaluated for their ability to recapitulate the experimental data: Rosetta $\Delta \Delta \mathrm{G}_{16}$, FoldX 17 , ELASPIC18, DeepDDG19, and PoPMuSiC.20 The crystal structure of BglB (PDB ID: 2JIE) was used across five different algorithms. First, using a previously described method 14 , the 2JIE structure was used as input to the Rosetta $\Delta \Delta \mathrm{G}$ application and run as previously described (SI 5). Briefly, 50 poses of the wild type and the mutant were generated for which 15 energy terms are reported from the score function used.16 The three lowest system energy scores out of 50 from WT and mutant were averaged to give the final Rosetta $\Delta \Delta \mathrm{G}$ score. 


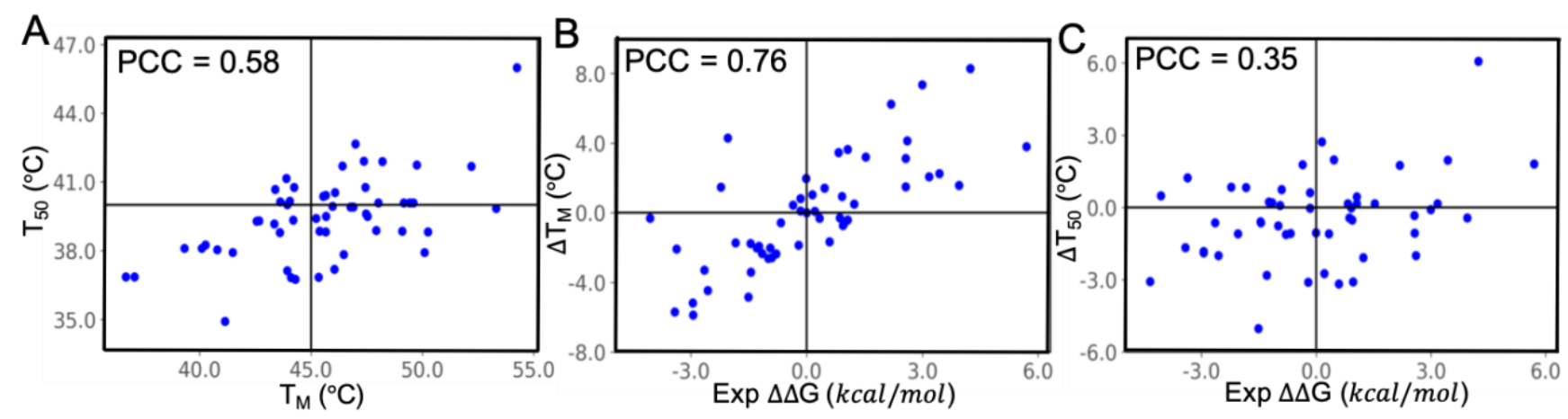

Figure 2. Comparison of two different experimental thermal stability dataset and experimentally-derived $\Delta \Delta \mathrm{G}$. (A) The relationship for each mutant between $\mathrm{T}_{50}$ and $\mathrm{T}_{\mathrm{M}}$. The PCC of 0.58 illustrates the two methods are modestly positively correlated with mutations that are in the extreme ends of the temperature range $\left( \pm 5^{\circ} \mathrm{C}\right)$ are generally in agreement. (B) The evaluation of $\Delta \mathrm{T}_{\mathrm{M}}$ with experimentally-derived $\Delta \Delta \mathrm{G}$ shows the two qualities are highly correlated $(\mathrm{PCC}=0.76)$ unlike $(\mathrm{C})$ the relationship between $\Delta \mathrm{T} 50$ and experimentally-derived $\Delta \Delta \mathrm{G}$ with a PCC of 0.35 .

Second, for the FoldX position-specific scoring metric (PSSM) protocol, the 2JIE structure was first minimized for any potential inaccurate rotamer assignment using the RepairPDB application. 17 The repaired PDB structure was mutated with single-point mutants and then modeled using FoldX PSSM. The model was scored based on 17 terms within FoldX force-field.17 Third, the ELASPIC protocol first constructs a homology model of the wild type using the crystal structure, sequence, molecular, and energetics information. Using the standard procedure described, FoldX algorithm was used to construct the mutant model. Combining the energetic information from FoldX along with 74 other features, Stochastic Gradient Boosting of Decision Trees are used to make the final prediction of the mutational change.18,21 Fourth, using a curated dataset derived from Protherm database, DeepDDG used their previously described Shared Residue Pair (SRP) neural network structure to make stability prediction. 19 Lastly, the fifth method evaluated is PoPMuSiC where the stability of the wild type structure and mutant were estimated using 13 statistical potential terms and additional two terms that count for the volume differences of the residues between WT and the mutant.20

A Pearson correlation (PCC) analysis was performed between the change in total system energy ( $\triangle \mathrm{TSE})$ of the five computational methods and the two thermal stability methods ( $\mathrm{T}_{\mathrm{M}}$ and $\mathrm{T}_{50}$ ). Additionally, the available individual features within the Rosetta $\Delta \Delta \mathrm{G}$ and FoldX PSSM force field were further evaluated against the TM dataset for correlation.

Finally, the $\triangle \mathrm{TSE}$ was also evaluated against isolated soluble protein and un-isolated insoluble protein for its statistical significance. Using the student T-test, the p-values for the five computational methods were obtained by treating the two categories between isolated soluble and insoluble protein as samples that are independent of each other with the variance being treated as unequal.

\section{RESULTS}

Evaluating the Relationship between $\mathbf{T}_{\mathbf{M}}$ and $\mathbf{T}_{\mathbf{5 0}}$. To the best of our knowledge, there has not been a large data set $(>50$ data points) directly comparing the $\mathrm{T}_{\mathrm{M}}$ and $\mathrm{T}_{50}$ relationship for a single set of protein mutants produced and characterized uniformly. It is important to distinguish both $\mathrm{T}_{\mathrm{M}}$ and $\mathrm{T}_{50}$ methods since the measurements are quantifying and reporting different structural and functional properties. $\mathrm{T}_{\mathrm{M}}$ is defined by the temperature at which half the enzyme is found in the unfolded state over folded state 12,22 and is often evaluated through denaturation assays, from which the thermodynamic measurements ( $\Delta$ Gunfolding) can be obtained.22 This method is generally a lower throughput method as purified protein is required to get an accurate measurement for the structural properties for the mutant being evaluated. T50 measures the temperature of half-inactivation that leads to irreversible unfolding 11,23 , and it is determined by the reduction of half of the enzymatic activity due heat-challenges.12 This is a very common assay for protein engineering due to its compatibility with high throughput assays and the ability to use cell lysates to evaluate function.

To complement our previously measured dataset of $\mathrm{T}_{50}, 51$ proteins were selected randomly and evaluated for Tм using the Protein Thermal Shift ${ }^{\mathrm{TM}}$ assay in order to compare $\mathrm{T}_{50}$ and $\mathrm{T}_{\mathrm{M}}$. The wild type $\mathrm{BglB} \mathrm{TM}_{\mathrm{M}}$ is $45.97 \pm 1.03{ }^{\circ} \mathrm{C}$, meanwhile the previously determined $\mathrm{T}_{50}$ is $39.9 \pm 0.1{ }^{\circ} \mathrm{C} .14$ When evaluating the entire data set, the $\mathrm{T}_{\mathrm{M}}$ range is between 37.1 and $54.3{ }^{\circ} \mathrm{C}$, slightly larger than what was observed for $\mathrm{T}_{50}$ which is between 34.9 and $46.0^{\circ} \mathrm{C}$ (SI 1-2). The highest $\mathrm{T}_{\mathrm{M}}$ in this data set is E167A, $54.3{ }^{\circ} \mathrm{C}\left(+8.33{ }^{\circ} \mathrm{C}\right)$, which is also observed to have a similar increase in $\mathrm{T}_{50}$ from wild type $\left(+6.06^{\circ} \mathrm{C}\right) .14$ The variant that has the lowest $\mathrm{T}_{\mathrm{M}}$ in this dataset is found to be E225A, with a $\Delta \mathrm{T}_{\mathrm{M}}$ of $-8.9^{\circ} \mathrm{C}$, which has corresponding $\mathrm{T}_{50}$ of $-3.1^{\circ} \mathrm{C}$.

The relationship between $\mathrm{T}_{50}$ and $\mathrm{T}_{\mathrm{M}}$ is plotted in Figure $2 \mathrm{~A}$. The Pearson coefficient correlation (PCC) of 0.58 shows the two methods are moderately positively correlated. Correlation between methods increased in cases where mutations resulted in extremely stable and unstable products, for example E167A and E225A, respectively. This is an expected result as for small changes $\left(<3{ }^{\circ} \mathrm{C}\right)$ in thermal stability; the differences in 

under aCC-BY-NC-ND 4.0 International license.

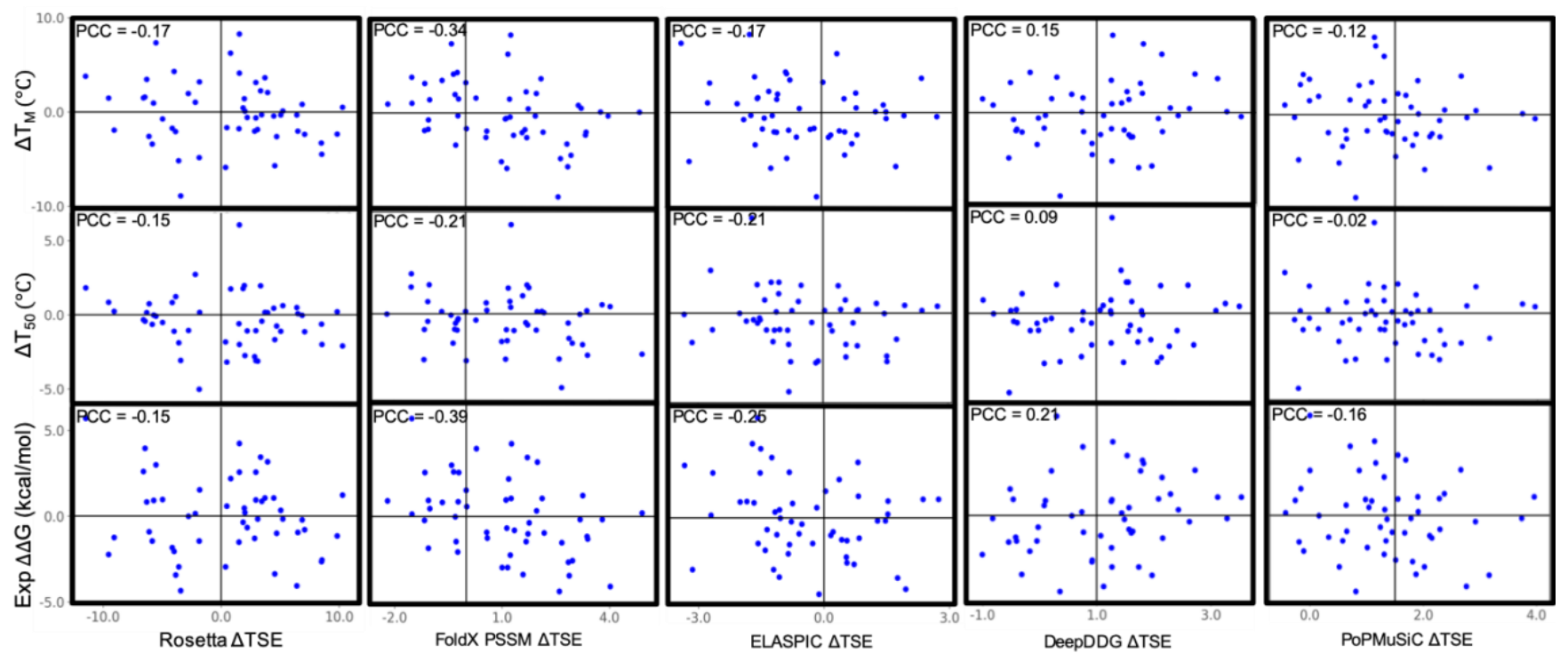

Figure 3. Evaluation of the five algorithms $\Delta \mathrm{TSE}$ versus the experimentally-derived $\Delta \Delta \mathrm{G}$, and $\mathrm{T}_{\mathrm{M}}$ and $\mathrm{T}_{50}$ dataset. The Pearson correlation for each performance against three types of experimental data was analyzed. No algorithm resulted in a significant correlation between the calculated energies and the observed $\mathrm{T}_{\mathrm{M}}$, $\mathrm{T}_{50}$, or $\Delta \Delta \mathrm{G}$ for this dataset.

Measurement methods would be expected to play a more significant role than for larger changes $\left(>5^{\circ} \mathrm{C}\right)$.

Evaluating computational stability tools using the BglB $\mathbf{T}_{M}$ dataset. The computational evaluation of protein stability of the current experimental $\mathrm{T}_{\mathrm{M}}$ dataset was analyzed in the same manner as our previous study did for T50.14 An energetically evaluated model for each mutant was generated using established computational methods, and subsequently plotted as a function of $\mathrm{T}_{\mathrm{M}}$ to evaluate the calculated energies related to the observed Тм. The PCC for the most commonly evaluated term, the difference in total system energy of the mutants versus the wild type protein $(\triangle \mathrm{TSE})$, was found to be highest for FoldX PSSM (PCC $=-0.34)$ with $\Delta \mathrm{T}_{\mathrm{M}}($ Fig 3$)$. Similarly, the FoldX PSSM correlations with experimentally-derived $\Delta \mathrm{T}_{50}$ data is found to be -0.21 . The overall relationship between the $\triangle \mathrm{TSE}$ and the current thermal stability dataset slightly improved for FoldX, DeepDDG, and PoPMuSiC, while Rosetta $\Delta \Delta \mathrm{G}$ and ELASPIC remained relatively unchanged with no significant correlation.

An analysis of each energetic term from Rosetta $\Delta \Delta G$ and FoldX PSSM did not uncover any specific parameter in either method's energetic evaluation that was strongly correlated with the $\mathrm{T}_{\mathrm{M}}$ data set, as was previously observed for the $\mathrm{T}_{50}$ data set 14 (SI 3). The strongest PCC for TM against any of the available energetic terms is 0.39 for the $\Delta$ backbone clash term from FoldX PSSM and -0.31 for the Omega energy term from Rosetta $\Delta \Delta \mathrm{G}$. Moreover, since the previous performance of the algorithms were evaluated using experimentally-derived $\Delta \Delta \mathrm{G}$ in $\mathrm{kcal} / \mathrm{mol}$, the same evaluation was done by converting the $\mathrm{T}_{\mathrm{M}}$ dataset into $\Delta \Delta \mathrm{G}$ (Fig 3). The PCC of -0.39 between experimental $\Delta \Delta \mathrm{G}$ and $\triangle \mathrm{TSE}$ for FoldX PSSM outperformed four other algorithms that were compared. The correlation between experimental $\Delta \Delta \mathrm{G}$ with $\Delta \mathrm{TSE}$ was not unexpected as $\mathrm{T}_{\mathrm{M}}$ is relatively correlated to $\Delta \Delta \mathrm{G}$ with a PCC of 0.76 (SI 1-3).

Based on this analysis, it is apparent that the general performance of all given methods only weakly correlates with the experimentally determined effects of the mutations. This data fails to support the hypothesis that the lack of a previously observed correlation of these established computational tools with observed changes in thermal stability in the BglB data set is due to the difference in the physical property being measured.

Protein expression prediction. The current data set consists primarily of modest thermal stability changes of $<5{ }^{\circ} \mathrm{C}$, calculated to be $\pm 4 \mathrm{kcal} / \mathrm{mol}$ of the native, and therefore may be challenging for current computational methods to predict. However, this change is only analyzing a fraction of the 129 mutants tested in the overall BglB data set. Of the 129 mutants, only 92 were found to be produced and isolated in a soluble form. As all purification procedures are conducted at room temperature, with the wild type $\mathrm{T}_{50}$ of $39.9{ }^{\circ} \mathrm{C}$, any reduction of $>18{ }^{\circ} \mathrm{C}$ would result in denatured, insoluble protein. Therefore, it seemed pertinent to evaluate if any of the five algorithms could differentiate variants in this data set that could be isolated as soluble protein versus those that were not able to be separated as soluble protein.

For this evaluation, all the previous 129 mutants reported were assessed using Rosetta $\Delta \Delta \mathrm{G}$, FoldX PSSM, ElASPIC, DeepDDG, and PoPMuSic in the same manner as carried out for $\mathrm{T}_{50}$ and $\mathrm{T}_{\mathrm{M}}$. A mutant was generally considered soluble if it was observed on an SDS-PAGE analysis and had an A280 of $>0.1 \mathrm{mg} / \mathrm{ml}$. The wild type protein produced using the methods described generally resulted in an average A280 of $1.5 \mathrm{mg} / \mathrm{ml}$, which would provide a $>10$-fold change in yield for mutants having an A280 less than $0.1 \mathrm{mg} / \mathrm{ml}$. While factors other than thermal stability can affect production and isolation of soluble protein, it is assumed in this case that the primary factor that decreases soluble protein yield is from denaturation of the mutant protein either during expression or purification. The results of this analysis are presented in Figure 4. 
bioRxiv preprint doi: https://doi.org/10.1101/856732; this version posted November 28, 2019. The copyright holder for this preprint (which was not certified by peer review) is the author/funder, who has granted bioRxiv a license to display the preprint in perpetuity. It is made available under aCC-BY-NC-ND 4.0 International license.

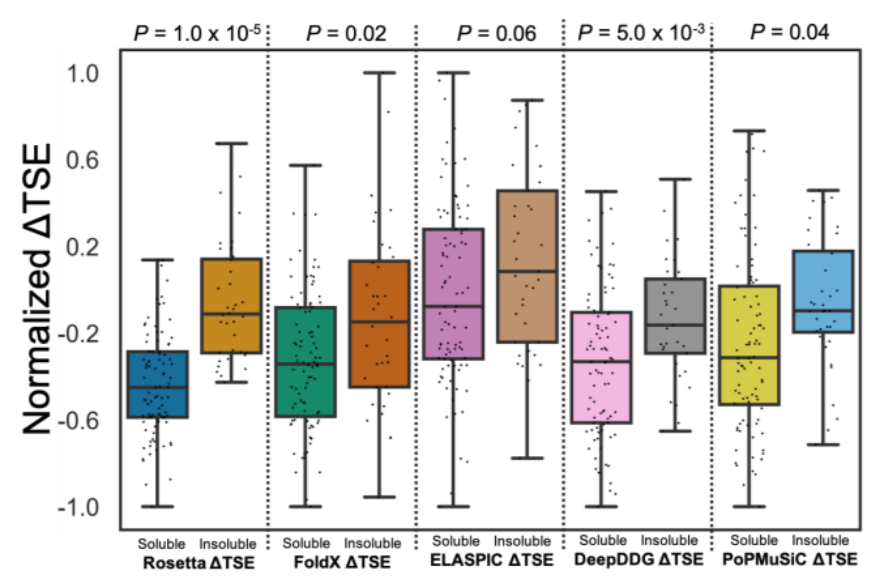

Figure 4. Computational prediction for the effect on mutant soluble protein production using five different algorithms. From left to right: Rosetta $\Delta \Delta \mathrm{G}$, FoldX PSSM, ELASPIC, DeepDDG, and PoPMuSiC of soluble and insoluble protein. In this case mutants that resulted in a significant (>10-fold) decrease in yield of purified soluble protein are considered insoluble. Significance in population differences is done using the Student's t-test.

Of the five algorithms evaluated, Rosetta $\Delta \Delta \mathrm{G}$, ELASPIC, and DeepDDG can capture the enrichment of mutants isolated as a soluble protein. The differences were evaluated for statistical significance using the student T-test, and the highest among the top three methods was shown in Rosetta $\Delta \Delta \mathrm{G}$ with a $p$-value of 1.0 x 10-5. In contrast, enrichment was lower for FoldX PSSM and PoPMuSiC with a $p$-value of 0.02 and 0.04 , respectively.

A few outliers were observed in all methods except for ELASPIC (SI 1-4). For example, the mutant G15N for both Rosetta $\Delta \Delta \mathrm{G}$ and FoldX PSSM was identified as severely energetically unfavorable, which is consistent with the observation that this variant was not able to be isolated as a soluble protein.

\section{DISCUSSION}

Both $\mathrm{T}_{\mathrm{M}}$ and $\mathrm{T}_{50}$ are methods commonly used to quantify different physical aspects of protein thermal stability, however there is relatively little information reported on the relationship of these two measurements. Using a data set of 51 protein mutants we observed that there is a moderate positive correlation (PCC of 0.58) between these two properties. Mutants with extreme stability changes, such as E164A $\left(>6^{\circ} \mathrm{C}\right)$, usually exhibit similar magnitude in $\mathrm{T}_{\mathrm{M}}$ and $\mathrm{T}_{50}$ results. However, the majority of the mutants exhibit a change of $3{ }^{\circ} \mathrm{C}$ or less in this $\mathrm{T}_{\mathrm{M}}$ and $\mathrm{T}_{50}$ data set being analyzed, in which range the effect of experimental variability may be significant, therefore analysis with larger data sets with more extreme stability changes may reveal an even stronger correlation of these two properties.

Consistent with our previous analysis, we found a lack of performance using established computational tools when predicting $\mathrm{T}_{\mathrm{M}}$ and $\mathrm{T}_{50}$ from the wild type for this data set. According to Jia et al, stability prediction using the experimentally-derived free energy change of unfolding $\Delta \Delta \mathrm{G}$ $(\Delta \mathrm{kcal} / \mathrm{mol})$ outperforms the prediction using $\Delta \mathrm{T}_{\mathrm{M}}(\Delta$ Celsius).24 However, in this case, we saw no significant change in predictive performance for all five computational tools when comparing to the experimentally-derived free energy change. In addition, we found that $T_{M}$ and $\Delta \Delta G$ to be strongly correlated with this data set, which may suggest that the improved performance is only relevant for more diverse datasets composed of different proteins as opposed to mutants of a single protein.

While none of the computational methods demonstrated a strong predictive power for the mutants in this study, Rosetta $\Delta \Delta \mathrm{G}$, FoldX PSSM, ELASPIC, and PoPMuSiC all have previously been shown to have high correlations with experimental data (PCC between 0.69 to 0.83).16,20,21,25 A major factor that could differentiate this study from previous work is that this data set has an experimental $\Delta \Delta \mathrm{G}$ range of $\pm \sim 4$ $\mathrm{kcal} / \mathrm{mol}$, which is significantly smaller than what the algorithms were usually trained on, which ranged from +8 to $5 \mathrm{kcal} / \mathrm{mol} .17$ This is further supported by the observation that Rosetta $\Delta \Delta \mathrm{G}$ does have a strong predictive performance for our data set when including mutants presumed to have large effects on $\mathrm{T}_{\mathrm{M}}$ and $\mathrm{T}_{50}$, because they failed to be isolated as soluble protein due to the substantial stability changes.

This study highlights the need for new computational tools that can more accurately predict modest changes in thermal stability, rather than major changes. This becomes particularly important because single-point mutants often increase thermal stability by a few degrees at a time, while major changes are more often produced from the synergistic effect of combining multiple mutations.11,26-28 Furthermore, as larger data sets of protein mutants with explicitly measured biophysical properties are generated, opportunities to explore combinations of molecular modeling and machine learning methods will become practical. These algorithms and data sets will enable the development of robust predictors of thermal stability.

\section{ASSOCIATED CONTENT}

\section{Supporting Information (SI)}

\section{SI 1-1. SDS-PAGE images for 51 BglB mutants and WT. (PDF)}

SI 1-2. A distribution analysis of temperatures observed for $\mathrm{T}_{M}$ and $\mathrm{T}_{50}$.

SI 1-3. PPC graph between $\Delta \mathrm{TM}$ and $\Delta \Delta \mathrm{G}$ of BglB mutants. (PDF)

SI 1-4. Evaluation of five computational methods on protein expression. (PDF)

SI 2. Images of $\mathrm{TM}_{\mathrm{M}}$ fluorescence graphs, derivative graphs, and Van't Hoff plot for 51 mutants and WT. (Zip)

SI 3. Rosetta $\Delta \Delta \mathrm{G}$ and FoldX PSSM correlations graphs with $\Delta \mathrm{TM}$ and experimental $\Delta \Delta \mathrm{G}$. Excel files of all the parameters from data acquisition. Excel files of the total system energy for DeepDDG, ELASPIC, and PoPMuSiC (Zip)

SI 4. Jupyter notebook for all thermal stability data acquisitions with all TM raw data files. (ipynb)

SI 5. Example files for Rosetta_ddg_monomer run. (Zip)

\section{AUTHOR INFORMATION}


bioRxiv preprint doi: https://doi.org/10.1101/856732; this version posted November $28,2019$. The copyright holder for this preprint (which was not certified by peer review) is the author/funder, who has granted bioRxiv a license to display the preprint in perpetuity. It is made available under aCC-BY-NC-ND 4.0 International license.

\section{Corresponding Author}

* Email: jbsiegel@ucdavis.edu

\section{Author Contributions}

Data curation: $\mathrm{PH}, \mathrm{SKSC}, \mathrm{HNF}$

Investigation: $\mathrm{PH}, \mathrm{SKSC}$

Methodology: PH, SKSC, MPC, RWC, JBS

Software: SKSC

Writing - original draft: $\mathrm{PH}$

Writing - review \& editing: PH, JBS, SKSC, MPC

\section{ACKNOWLEDGMENT}

This work was supported by the University of California Davis, the National Institutes of Health [R01 GM 076324-11], the

National Science Foundation [Award Numbers 1827246 , 1805510, 1627539], and the National Institute of Environmental Health Sciences of the National Institutes of Health [Award Number P42ES004699]. The content is solely the responsibility of the authors and does not necessarily represent the official views of the National Institutes of Health, National Institute of Environmental Health Sciences, National Science Foundation, or UC Davis.

\section{REFERENCES}

(1) Iyer, P. V.; Ananthanarayan, L. Enzyme Stability and Stabilization-Aqueous and Non-Aqueous Environment. Process Biochem. 2008, 43 (10), 1019-1032.

https://doi.org/10.1016/j.procbio.2008.06.004.

(2) Turner, P.; Mamo, G.; Karlsson, E. N. Potential and Utilization of Thermophiles and Thermostable Enzymes in Biorefining. Microb. Cell Fact. 2007, 6, 1-23. https://doi.org/10.1186/1475-2859-6-9.

(3) Ferdjani, S.; Ionita, M.; Roy, B.; Dion, M.; Djeghaba, Z.; Rabiller, C.; Tellier, C. Correlation between Thermostability and Stability of Glycosidases in Ionic Liquid. Biotechnol. Lett. 2011, 33 (6), 1215-1219. https://doi.org/10.1007/s10529-011-0560-5.

(4) Xie, Y.; An, J.; Yang, G.; Wu, G.; Zhang, Y.; Cui, L.; Feng, Y. Enhanced Enzyme Kinetic Stability by Increasing Rigidity within the Active Site. J. Biol. Chem. 2014, 289 (11), 7994-8006. https://doi.org/10.1074/jbc.M113.536045.

(5) Wu, I.; Arnold, F. H. Engineered Thermostable Fungal Cel6A and Cel7A Cellobiohydrolases Hydrolyze Cellulose Efficiently at Elevated Temperatures. Biotechnol. Bioeng. 2013, 110 (7), 1874-1883. https://doi.org/10.1002/bit.24864.

(6) Kazlauskas, R. J.; Bornscheuer, U. T. Finding Better Protein Engineering Strategies. Nat. Chem. Biol. 2009, 5, 526.

(7) Denard, C. A.; Ren, H.; Zhao, H. Improving and Repurposing Biocatalysts via Directed Evolution. Curr. Opin. Chem. Biol. 2015, 25, 55-64. https://doi.org/10.1016/j.cbpa.2014.12.036.

Borgo, B.; Havranek, J. J. Automated Selection of Stabilizing Mutations in Designed and Natural Proteins. Proc. Natl. Acad. Sci. 2012, 109 (5), 1494 1499. https://doi.org/10.1073/pnas.1115172109.

Jacak, R.; Leaver-Fay, A.; Kuhlman, B. Computational Protein Design with Explicit Consideration of Surface Hydrophobic Patches. Proteins Struct. Funct. Bioinforma. 2012, 80 (3), 825-838. https://doi.org/10.1002/prot.23241.

(10) Lehmann, M.; Loch, C.; Middendorf, A.; Studer, D.; Lassen, S. F.; Pasamontes, L.; van Loon, A. P. G. M.; Wyss, M. The Consensus Concept for Thermostability Engineering of Proteins: Further Proof of Concept. Protein Eng. 2002, 15 (5), 403411.

Goldenzweig, A.; Fleishman, S. J. Principles of Protein Stability and Their Application in Computational Design. Annu. Rev. Biochem. 2018, 87 (1), 105-129. https://doi.org/10.1146/annurevbiochem-062917-012102.

(12) Polizzi, K. M.; Bommarius, A. S.; Broering, J. M.; Chaparro-Riggers, J. F. Stability of Biocatalysts. Current Opinion in Chemical Biology. 2007. https://doi.org/10.1016/j.cbpa.2007.01.685.

(13) Bloom, J. D.; Labthavikul, S. T.; Otey, C. R.; Arnold, F. H. Protein Stability Promotes Evolvability. Proc. Natl. Acad. Sci. 2006, 103 (15), 5869-5874.

https://doi.org/10.1073/pnas.0510098103.

(14) Carlin, D. A.; Hapig-Ward, S.; Chan, B. W.; Damrau, N.; Riley, M.; Caster, R. W.; Bethards, B.; Siegel, J. B. Thermal Stability \& Kinetic Constants for 129 Variants of a Family 1 Glycoside Hydrolase Reveal That Enzyme Activity \& Stability Can Be Separately Designed. PLoS One 2017, 12 (5), 1-13. https://doi.org/10.1371/journal.pone.0176255.

(15) Schrödinger, L. The PyMOL Molecular Graphics System http://www.pymol.org.

(16) Kellogg, E. H.; Leaver-Fay, A.; Baker, D. Changes in Protein Structure and Stability. Proteins 2011, 79 (3), $830-838$. https://doi.org/10.1002/prot.22921.Role.

Schymkowitz, J.; Borg, J.; Stricher, F.; Nys, R.; Rousseau, F.; Serrano, L. The FoldX Web Server: An Online Force Field. Nucleic Acids Res. 2005, 33, 382-388. https://doi.org/10.1093/nar/gki387.

(18) Witvliet, D. K.; Strokach, A.; Giraldo-Forero, A. F.; Teyra, J.; Colak, R.; Kim, P. M. ELASPIC WebServer: Proteome-Wide Structure-Based Prediction 
bioRxiv preprint doi: https://doi.org/10.1101/856732; this version posted November $28,2019$. The copyright holder for this preprint (which was not certified by peer review) is the author/funder, who has granted bioRxiv a license to display the preprint in perpetuity. It is made available under aCC-BY-NC-ND 4.0 International license.

of Mutation Effects on Protein Stability and Binding Affinity. Bioinformatics 2016, 32 (10), 1589-1591. https://doi.org/10.1093/bioinformatics/btw031.

(19) Cao, H.; Wang, J.; He, L.; Qi, Y.; Zhang, J. Z. DeepDDG: Predicting the Stability Change of Protein Point Mutations Using Neural Networks. $J$. Chem. Inf. Model. 2019, 59 (4), 1508-1514. https://doi.org/10.1021/acs.jcim.8b00697.

(20) Dehouck, Y.; Kwasigroch, J. M.; Gilis, D.; Rooman, M. PoPMuSiC 2.1: A Web Server for the Estimation of Protein Stability Changes upon Mutation and Sequence Optimality. BMC Bioinformatics 2011, 12 (1), 151. https://doi.org/10.1186/1471-2105-12-151.

(21) Berliner, N.; Teyra, J.; Çolak, R.; Lopez, S. G.; Kim, P. M. Combining Structural Modeling with Ensemble Machine Learning to Accurately Predict Protein Fold Stability and Binding Affinity Effects upon Mutation. PLoS One 2014, 9 (9). https://doi.org/10.1371/journal.pone.0107353.

(22) Musil, M.; Konegger, H.; Hon, J.; Bednar, D.; Damborsky, J. Computational Design of Stable and Soluble Biocatalysts. ACS Catal. 2019, 9 (2), 10331054. https://doi.org/10.1021/acscatal.8b03613.

(23) Colón, W.; Church, J.; Sen, J.; Thibeault, J.; Trasatti, H.; Xia, K. Biological Roles of Protein Kinetic Stability. Biochemistry 2017, 56 (47), 6179-6186. https://doi.org/10.1021/acs.biochem.7b00942.
(24)

Jia, L.; Yarlagadda, R.; Reed, C. C. Structure Based Thermostability Prediction Models for Protein Single Point Mutations with Machine Learning Tools. PLoS One 2015, 10 (9), 1-19. https://doi.org/10.1371/journal.pone.0138022.

(25) Guerois, R.; Nielsen, J. E.; Serrano, L. Predicting Changes in the Stability of Proteins and Protein Complexes: A Study of More than 1000 Mutations. J. Mol. Biol. 2002, 320 (2), 369-387. https://doi.org/10.1016/S0022-2836(02)00442-4.

(26) Korkegian, A; Black, ME; Baker, D; Stoddard, B. Computational Thermostabilization of an Enzyme. 2005, 308 (5723), 857-860. https://doi.org/10.1126/science.1107387.

(27) Wakabayashi, H.; Griffiths, A. E.; Fay, P. J. Combining Mutations of Charged Residues at the A2 Domain Interface Enhances Factor VIII Stability over Single Point Mutations. 2009, 7 (3), 492-495. https://doi.org/10.1111/j.15387836.2008.03256.x.Combining.

(28) Li, Guanlin; Fang, Xingrong; Su, Feng; Chen, Yuan; $\mathrm{Xu}, \mathrm{Li}$; Yan, Y. Enhancing the Thermostability of Rhizomucor Miehei Lipase with a Limited Screening Library by Rational-Design Point Mutations and Disulfide Bonds. 2018, 84 (2), 1-16. https://doi.org/10.1128/AEM.02129-17. 